\section{Accuracy, diagnostische}

R.-D. Hilgers ${ }^{1}$, N. Heussen ${ }^{1}$ und S. Stanzel ${ }^{2}$

${ }^{1}$ Institut für Medizinische Statistik, Universitätsklinikum der RWTH Aachen, Aachen, Deutschland

${ }^{2}$ Heidelberg, Deutschland

Synonym(e) Diagnostische Richtigkeit

Englischer Begriff accuracy of a diagnostic test

Definition Beschreibt die Fähigkeit eines diagnostischen Testverfahrens, zwischen 2 alternativen Gesundheitszuständen zu unterscheiden.

Beschreibung Diagnostische Accuracy ist die grundlegende Eigenschaft eines diagnostischen Tests $(\triangleright$ Test, diagnostischer). Zweig und Campbell (1993) definieren „,diagnostische Accuracy“ als die Fähigkeit, zwischen 2 Subklassen von Individuen unterscheiden zu können, wenn dies klinisch notwendig erscheint. Diese Definition zielt auf die Qualität der
Information $\mathrm{ab}$ und ist $\mathrm{zu}$ unterscheiden vom Aspekt des praktischen Nutzens der Information ( $\triangleright$ Usefulness). Beides sind Aspekte zur Bewertung der Leistungsfähigkeit des Tests, wobei am Anfang die Bewertung der diagnostischen Accuracy steht.

Vielfach wird die diagnostische Accuracy (Richtigkeit) durch den relativen Anteil korrekter Testentscheidungen beschrieben. Die diagnostische Accuracy wird üblicherweise geschätzt durch den Quotienten

$$
\frac{a+d}{a+b+c+d}
$$

(Bezeichnungen s. Tabelle im Stichwort $\triangleright$ Vierfeldertafel).

\section{Literatur}

Zweig MH, Campbell G (1993) Receiver-operating characteristic (ROC) plots: a fundamental evaluation tool in clinical medicine. Clin Chem 39:561-577 